\title{
Room temperature spin filtering effect in GaNAs: Role of hydrogen
}

\author{
Yuttapoom Puttisong, Daniel Dagnelund, Irina Buyanova, C W Tu, A Polimeni, \\ M Capizzi and Weimin Chen
}

\section{Linköping University Post Print}

N.B.: When citing this work, cite the original article.

Original Publication:

Yuttapoom Puttisong, Daniel Dagnelund, Irina Buyanova, C W Tu, A Polimeni, M Capizzi and Weimin Chen, Room temperature spin filtering effect in GaNAs: Role of hydrogen, 2011, Applied Physics Letters, (99), 15, 152109.

http://dx.doi.org/10.1063/1.3651761

Copyright: American Institute of Physics (AIP) http://www.aip.org/

Postprint available at: Linköping University Electronic Press

http://urn.kb.se/resolve?urn=urn:nbn:se:liu:diva-72139 


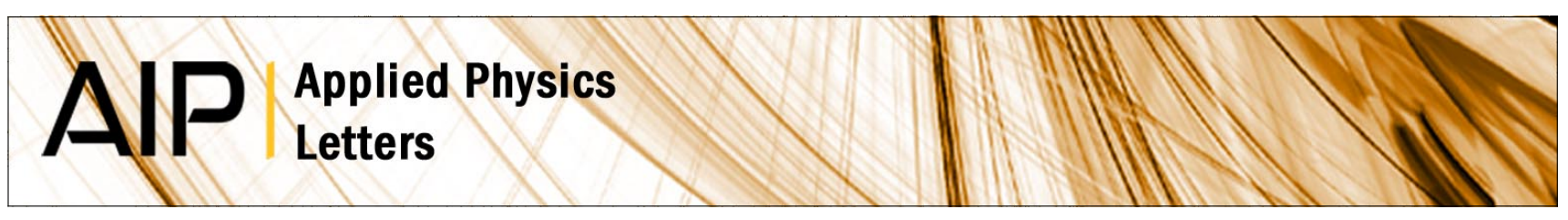

\section{Room temperature spin filtering effect in GaNAs: Role of hydrogen}

Y. Puttisong, D. Dagnelund, I. A. Buyanova, C. W. Tu, A. Polimeni et al.

Citation: Appl. Phys. Lett. 99, 152109 (2011); doi: 10.1063/1.3651761

View online: http://dx.doi.org/10.1063/1.3651761

View Table of Contents: http://apl.aip.org/resource/1/APPLAB/v99/i15

Published by the American Institute of Physics.

\section{Related Articles}

Optically controlled spin polarization in a spin transistor

Appl. Phys. Lett. 94, 162109 (2009)

High temperature electron spin relaxation in bulk GaAs

Appl. Phys. Lett. 93, 132112 (2008)

Nuclear and excitonic spin polarization formed using cross-linearly polarized pulse pair via half-localized state in a single self-assembled quantum dot

J. Appl. Phys. 103, 103530 (2008)

Optically spin oriented electron transmission across fully epitaxial $\mathrm{Fe} 304 / \mathrm{GaAs}(001)$ interfaces

J. Appl. Phys. 103, 07D705 (2008)

Spin-valve photodiode

Appl. Phys. Lett. 83, 3737 (2003)

\section{Additional information on Appl. Phys. Lett.}

Journal Homepage: http://apl.aip.org/

Journal Information: http://apl.aip.org/about/about_the_journal

Top downloads: http://apl.aip.org/features/most_downloaded

Information for Authors: http://apl.aip.org/authors

\section{ADVERTISEMENT}

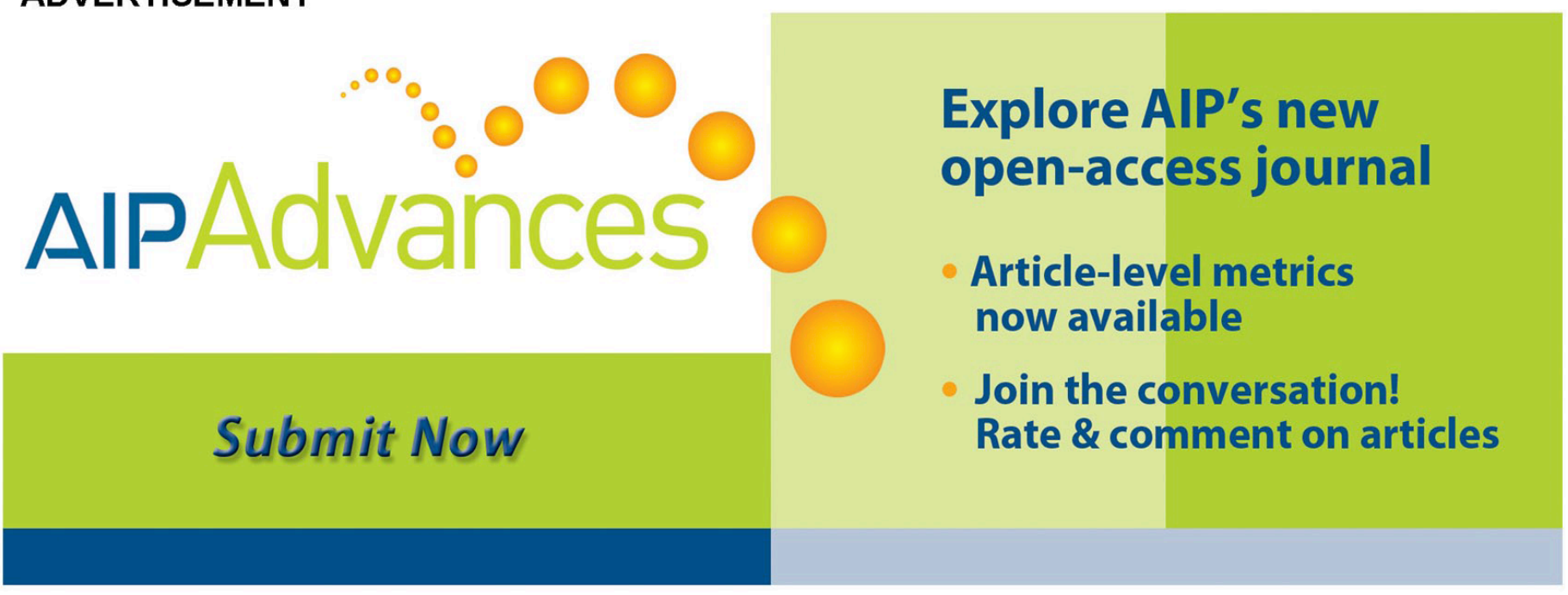




\title{
Room temperature spin filtering effect in GaNAs: Role of hydrogen
}

\author{
Y. Puttisong, ${ }^{1}$ D. Dagnelund, ${ }^{1}$ I. A. Buyanova, ${ }^{1}$ C. W. Tu, ${ }^{2}$ A. Polimeni, ${ }^{3}$ M. Capizzi, ${ }^{3}$ \\ and W. M. Chen ${ }^{1, a)}$ \\ ${ }^{1}$ Department of Physics, Chemistry and Biology, Linköping University, S-581 83 Linköping, Sweden \\ ${ }^{2}$ Department of Electrical and Computer Engineering, University of California, La Jolla, \\ California 92093, USA \\ ${ }^{3}$ INFM and Dipartimento di Fisica, Università di Roma "La Sapienza," Piazzale A. Moro 2, \\ I-00185 Roma, Italy
}

(Received 23 September 2011; accepted 25 September 2011; published online 13 October 2011)

\begin{abstract}
Effects of hydrogen on the recently discovered defect-engineered spin filtering in GaNAs are investigated by optical spin orientation and optically detected magnetic resonance. Post-growth hydrogen treatments are shown to lead to nearly complete quenching of the room-temperature spin-filtering effect in both GaNAs epilayers and GaNAs/GaAs multiple quantum wells, accompanied by a reduction in concentrations of $\mathrm{Ga}_{\mathrm{i}}$ interstitial defects. Our finding provides strong evidence for efficient hydrogen passivation of these spin-filtering defects, likely via formation of complexes between $\mathrm{Ga}_{\mathrm{i}}$ defects and hydrogen, as being responsible for the observed strong suppression of the spin-filtering effect after the hydrogen treatments. (C) 2011 American Institute of Physics. [doi:10.1063/1.3651761]
\end{abstract}

Semiconductor spintronics is currently one of the most hotly pursued research fields, which explores the electron spin for future electronics, photonics, and quantum information technology. ${ }^{1-5}$ Despite of impressive progresses during recent years, many key issues are still unresolved. Among many challenges, generation of strongly spin-polarized electrons in semiconductors at room temperature (RT) remains difficult. Recently, an approach by exploiting the defect-engineered spin filtering effect has been proposed that is capable of creating strong electron spin polarization $\left(P_{e}>30 \%\right)$ in non-magnetic $\mathrm{Ga}$ (In)NAs alloys at RT. ${ }^{6-9}$ This is accomplished by utilizing spin-dependent recombination (SDR) via spin-polarized paramagnetic defects, which selectively filters out conduction electrons with spin orientation opposite to that of the defect electron while keeping intact those with the same spin orientation due to spin blockade. Such spin-filtering effect was also shown to turn GaNAs into an efficient RT spin detector, which paves the way for in-depth studies of RT spin injection and spin loss processes in the related material systems. ${ }^{10}$

To further improve the efficiency of the defectengineered spin-filtering effect, many key material parameters and physical processes must be identified, understood, and optimized. Previous studies have shown that the spin-filtering effect critically depends on $\mathrm{N}$ composition and growth temperature of $\mathrm{Ga}(\mathrm{In}) \mathrm{NAs}$ alloys, as well as post-growth thermal annealing. 6 It was further demonstrated that the dominant spin-filtering defects, i.e., $\mathrm{Ga}_{\mathrm{i}}$-interstitial defects, are common grown-in defects in alloys prepared by many modern epitaxial techniques including gas-source molecular beam epitaxy (GS$\mathrm{MBE}$ ), solid-source MBE, and metal-organic chemical vapor deposition. ${ }^{11}$ Up to now, however, the role of hydrogen in the spin-filtering remains unknown. The main interest in this issue is two-fold. First, hydrogen is known to be a fast diffuser and chemically active. It can be introduced as a common residual impurity during growth and processing of many semiconduc-

${ }^{\text {a)} E l e c t r o n i c ~ m a i l: ~ w m c @ i f m . l i u . s e . ~}$ tors. $^{12}$ As the growth processes of $\mathrm{Ga}(\mathrm{In}) \mathrm{NAs}$ by, e.g., GSMBE typically involve hydrogen, ${ }^{13,14}$ an obvious question is if hydrogen atoms are involved in the spin-filtering defects, e.g., as a part of the $\mathrm{Ga}_{\mathrm{i}}$ complexes. Such involvement would provide a possible explanation for the observed reduction and transformation of the grown-in $\mathrm{Ga}_{\mathrm{i}}$ complexes during postgrowth thermal annealing when hydrogen atoms became mobile and could be dissociated from the $\mathrm{Ga}_{\mathrm{i}}$ complexes. ${ }^{6}$ The second reason for the interest in the role of hydrogen in the spin filtering stems from the fascinating effect of hydrogen in passivating $\mathrm{N}$ and thereby tuning the bandgap energy of the alloys, observed after post-growth hydrogen treatments. ${ }^{15-18}$ Remarkable improvement in radiative recombination efficiency at RT was also demonstrated and was attributed to hydrogen passivation of non-radiative recombination defect centers. ${ }^{18}$ Possible involvement of hydrogen in the spinfiltering defects can provide an interesting prospect to combine bandgap engineering ${ }^{19}$ with spin engineering for optimal device applications. In this work, we aim to examine and understand the effect of post-growth hydrogen treatments on the defect-engineered spin filtering in GaNAs alloys.

Two representative structures were studied in this work: (1) a $\mathrm{GaN}_{0.013} \mathrm{As}_{0.987}$ epilayer with a thickness of $1100 \AA$ and (2) 7 periods of $\mathrm{GaN}_{0.016} \mathrm{As}_{0.984} / \mathrm{GaAs}(50 \AA / 200 \AA)$ multi-quantum wells (MQWs). They were grown at $420^{\circ} \mathrm{C}$ on a (001) GaAs substrate by GS-MBE. The post-growth hydrogen treatments were done at $300^{\circ} \mathrm{C}$ by using a Kaufmann source, with a dose of $10^{18}$ ions $/ \mathrm{cm}^{2}$. Photoluminescence (PL) experiments were performed at RT in a back scattering configuration. A tunable Ti-sapphire laser was used as an excitation source, with excitation photon energy above the GaNAs bandgap (at 780-980 nm). Circular polarization of the excitation beam in optical spin orientation experiments $^{20}$ was produced by a $\lambda / 4$-plate in combination with a linear polarizer. PL emissions were dispersed by a grating monochromator and detected by a cooled Ge detector. The degree of circular polarization of PL 
$\left(P_{P L}=\left(I_{\sigma^{+}}-I_{\sigma^{-}}\right) /\left(I_{\sigma^{+}}+I_{\sigma^{-}}\right)\right)$was monitored by using a photoelastic modulator together with a linear polarizer. Optically detected magnetic resonance (ODMR) measurements were performed at $2 \mathrm{~K}$ at $9.214 \mathrm{GHz}$. The spin filtering effect was monitored in the following two ways ${ }^{6-10}$ : (1) the ratio between total PL intensity under circularly and linearly polarized excitation, denoted as an SDR ratio $I^{\sigma^{+}} / I^{\sigma^{x}}$; (2) $P_{P L}$ under a given circularly polarized excitation (either $\sigma^{+}$ or $\sigma^{-}$).

RT PL spectra from the as-grown samples are shown in the upper panels of Figs. 1(a) and 1(b), obtained under $\sigma^{x}$ or $\sigma^{+}$excitation. They are dominated by a broad PL band arising from band-to-band (BB) transitions between conduction band $(\mathrm{CB})$ electrons and valence band (VB) holes, which consist of the CB-lh (light-hole) BB at the longer wavelength and the CB-hh (heavy-hole) BB at the shorter wavelength. ${ }^{6-9}$ Due to their strong overlap, however, the two components cannot be resolved in the PL spectra. Both as-grown epilayer and MQWs exhibit a sizable SDR ratio (about 1.6), displayed in the upper panel of Figs. 1(a) and 1(b), indicating active spin-filtering effect. ${ }^{21}$ The strong $\mathrm{CB} P_{e}$ as a result of the spin filtering effect is directly reflected by the observed strong $P_{P L}$ (up to $25 \%$ ) under $\sigma^{+}$excitation, shown in the lower panel of Figs. 1(a) and 1(b). The observed spectral dependence of $P_{P L}$ is due to the fact that $P_{P L}$ of the CB-hh and CB-lh transitions with a given $P_{e}$ are expected to be co- and counter-polarized with the excitation light, respectively. ${ }^{22}$

To confirm that the observed strong $P_{P L}$ is indeed induced by $\mathrm{CB} P_{e}$ created in optical spin orientation under circularly polarized excitation, we have measured $P_{P L}$ under $\sigma^{x}$ excitation, and the results are also shown in the lower panel of Figs. 1(a) and 1(b). No $P_{P L}$ was observed in this case, as expected when no $P_{e}$ can be created by $\sigma^{x}$ excitation.
After post-growth hydrogen treatments, the PL bands from both epilayer and MQWs display a blue shift [Figs. 1(c) and 1(d)] known to be induced by the $\mathrm{H}$ passivation of $\mathrm{N}$ and its associated alloying effect. ${ }^{15-19}$ Strikingly, the spin filtering effect is strongly suppressed after the hydrogen treatments, resulting in complete quenching of $P_{P L}$ and also disappearance in enhancement of the SDR ratio under $\sigma^{+}$excitation. In view of a strong dependence of excitation density on the spin-filtering effect from earlier studies, ${ }^{6-10}$ a systematic study of the SDR ratio as a function of excitation densities was carried out to verify that the vanishing SDR effect was not just valid under a specific excitation condition used. The representative results are shown in Fig. 2, taken the GaNAs epilayer as an example, which confirm that the observed vanishing of the spin-filtering effect after hydrogen treatment is valid regardless of excitation density. By analyzing the excitation-density dependence of the SDR ratio with the aid of coupled rate equations, ${ }^{6-10}$ we are able to estimate the relative change in the concentrations of the spin-filtering defects. It yields $N_{c}^{H} \leq 0.14 N_{c}^{A G}$, revealing a significant reduction of the spin-filtering defects after the hydrogen treatments down to less than $14 \%$ of the original value in the as-grown sample. Here, $N_{c}^{A G}$ and $N_{c}^{H}$ denote the concentrations of the spin-filtering defects in the as-grown and hydrogenated sample, respectively.

To confirm the suggested decrease in the concentrations of the spin-filtering defects, we performed ODMR studies that have earlier identified $\mathrm{Ga}_{\mathrm{i}}$ defects as the dominant spinfiltering defects in as-grown and annealed GaNAs. ${ }^{6,11}$ Typical ODMR spectra are shown in Fig. 3(a), taken as an example the GaNAs epilayer. In the as-grown GaNAs, rather strong ODMR signals were observed. A detailed analysis of the ODMR spectra by an effective spin Hamiltonian ${ }^{6}$ reveals
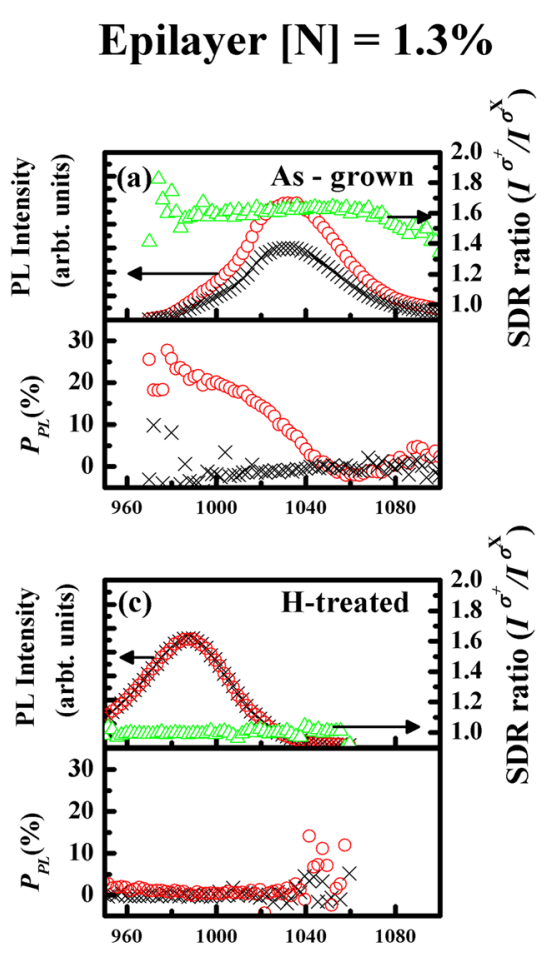

Wavelength (nm)

\section{MQWs $[N]=1.6 \%$}

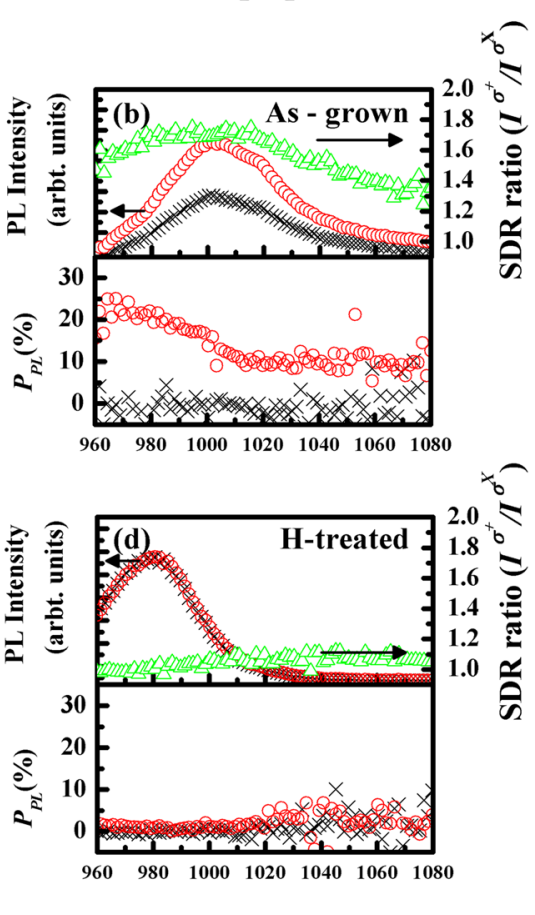

Wavelength (nm)
FIG. 1. (Color online) PL and polarization spectra at RT from the as-grown (a) GaNAs epilayer and (b) GaNAs MQWs. Their corresponding spectra after the hydrogen treatments are shown in (c) and (d). The crosses and open circles represent the PL (the upper panels) and $P_{P L}$ (the lower panels) spectra obtained under $\sigma^{x}$ and $\sigma^{+}$excitation, respectively. The open triangles denote the measured SDR ratios. All data were obtained under the excitation photon wavelength of $920 \mathrm{~nm}$, at a power of $300 \mathrm{~mW}$ for (a) and (c) and 350 $\mathrm{mW}$ for (b) and (d). 


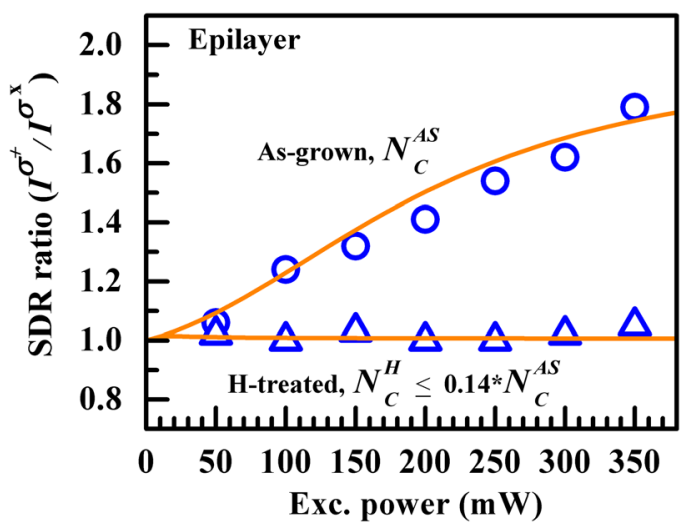

FIG. 2. (Color online) SDR ratios as a function of excitation power obtained at RT from the as-grown (the open circles) and hydrogen-treated (the open triangles) GaNAs epilayer. The SDR ratios were taken at the peak positions of the PL emissions under the excitation at $920 \mathrm{~nm}$. The solid lines are the simulation curves from the rate equations analysis, yielding the specified relative concentrations of the involved spin-filtering defects.

the presence of two types of $\mathrm{Ga}_{\mathrm{i}}$ defects with different hyperfine parameters (A) and electron g-factors $(\mathrm{g})$, namely $\mathrm{Ga}_{\mathrm{i}}-\mathrm{A}$ and $\mathrm{Ga}_{\mathrm{i}}-\mathrm{B},{ }^{11}$ together with a spin- $-1 / 2$ defect of unknown origin with $g=2.04$. The simulated ODMR spectra based on the spin Hamiltonian parameters for these defects ${ }^{11}$ are displayed in Fig. 3(b). The strong suppression of the ODMR signals after the hydrogen treatments [Fig. 3(a)] indicates a significant reduction in the role of these defects in carrier recombination and thus in their concentrations. ${ }^{6,23}$ This finding provides strong evidence for hydrogen passivation of these defects as the cause for the observed quenching of the spin filtering effect. Another piece of evidence for the hydrogen passivation effect is an increase in the RT PL intensity under $\sigma^{x}$ excitation after the hydrogen treatments, which can be interpreted by passivation of the spin-filtering defects that were known to be the dominant non-radiative recombination centers in $\mathrm{Ga}$ (In)NAs alloys. ${ }^{11}$ The passivation of the $\mathrm{Ga}_{\mathrm{i}}$ defects in GaNAs is likely due to formation of complexes

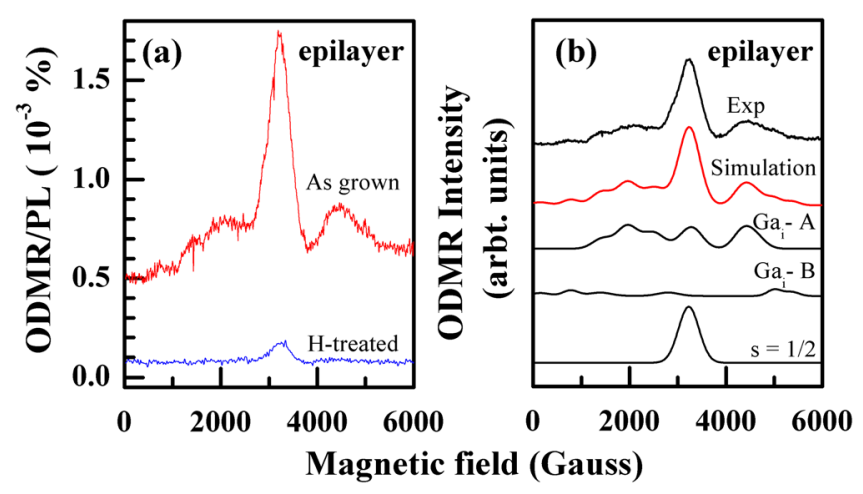

FIG. 3. (Color online) (a) ODMR spectra obtained by monitoring total BB PL intensity from the as-grown and hydrogen-treated GaNAs epilayer. The ODMR intensities are normalized by total PL intensity. (b) The simulated ODMR spectrum of the as-grown GaNAs epilayer, including the contributions from two $\mathrm{Ga}_{\mathrm{i}}$ defects (i.e., $\mathrm{Ga}_{\mathrm{i}} \mathrm{A}$ and $\mathrm{Ga}_{\mathrm{i}} \mathrm{B}$ ) and an unknown $\mathrm{S}=1 / 2$ defect (a single line with $g=2.04)$, calculated from the spin Hamiltonian ${ }^{6}$ with the following fitting parameters: $g=2.00 \quad$ (1.99) and $A\left({ }^{69} \mathrm{Ga}\right)=745 \times 10^{-4} \mathrm{~cm}^{-1}\left(1250 \times 10^{-4} \mathrm{~cm}^{-1}\right)$ for $\mathrm{Ga}_{\mathrm{i}}-\mathrm{A}\left(\mathrm{Ga}_{\mathrm{i}} \mathrm{B}\right)$. The sign of the ODMR signals is negative, corresponding to a decrease of PL intensity upon spin resonant transitions. between the original defects and $\mathrm{H}$, thereby removing the active role of the $\mathrm{Ga}_{\mathrm{i}}$ defects in SDR and spin filtering.

In summary, we have studied the effect of post-growth hydrogen treatments on the defect-engineered spin-filtering in GaNAs. We have shown that the spin-filtering effect is completely vanished after the hydrogen incorporation in both GaNAs epilayer and GaNAs/GaAs MQWs. We have provided experimental evidence for the hydrogen passivation of the spin-filtering defects as the dominant mechanism responsible for this finding. Our results also indicate that hydrogen is unlikely to be a part of the spin-filtering defects in the as-grown samples, despite of the fact that $\mathrm{H}$ is commonly present during the growth.

${ }^{1}$ S. A. Wolf, D. D. Awschalom, R. A. Buhrman, J. M. Daughton, S. von Molnár, M. L. Roukes, A. Y. Chtchelkanova, and D. M. Treger, Science 294, 1488 (2001).

${ }^{2}$ I. Žutić, J. Fabian, and S. Das Sarma, Rev. Mod. Phys. 76, 323 (2004).

${ }^{3}$ D. D. Awaschalom and M. F. Flatté, Nat. Phys. 3, 153 (2007).

${ }^{4}$ Spintronics, Semiconductors and Semimetals, edited by T. Dietl, D. D. Awschalom, M. Kaminska, and H. Ohno (Academic, New York, 2008), Vol. 82.

${ }^{5}$ Handbook of Spintronic Semiconductors, edited by W. M. Chen and I. A. Buyanova (Pan Stanford, Singapore, 2010).

${ }^{6}$ X. J. Wang, I. A. Buyanova, F. Chao, D. Zhao, D. Lagarde, A. Balocchi, X. Maries, C. W. Tu, J. C. Harmand, and W. M. Chen, Nature Mater. 8, 198 (2009).

${ }^{7}$ V. K. Kalevich, E. L. Ivchenko, M. M. Afanasiev, A. Yu. Shiryaev, A. Yu. Egorov, V. M. Ustinov, and Y. Masumoto, JETP Lett. 82, 455 (2005).

${ }^{8}$ D. Lagarde, L. Lombez, X. Marie, A. Balocchi, T. Amand, V. K. Kalevich, A. Shiryaev, E. Ivechenko, and A. Egorov, Phys. Stat. Sol. (a) 204, 208 (2007).

${ }^{9}$ Y. Puttisong, X. J. Wang, I. A. Buyanova, H. Carrére, F. Zhao, A. Balocchi, X. Marie, C. W. Tu, and W. M. Chen, Appl. Phys. Lett. 96, 052104 (2010).

${ }^{10}$ Y. Puttisong, X. J. Wang, I. A. Buyanova, C. W. Tu, L. Geelhaar, H. Riechert, and W. M. Chen, Appl. Phys. Lett. 98, 012112 (2011).

${ }^{11}$ X. J. Wang, Y. Puttisong, C. W. Tu, A. J. Ptak, V. K. Kalevich, A. Yu Egorov, L. Geelhaar, H. Riechert, W. M. Chen, and I. A. Buyanova, Appl. Phys. Lett. 95, 241904 (2009).

${ }^{12}$ S. J. Pearton, Int. J. Mod. Phys. B 8, 1247 (1994).

${ }^{13}$ H. P. Xin, C. W. Tu, and M. Geva, Appl. Phys. Lett. 75, 1416 (1999).

${ }^{14}$ H. P. Xin, C. W. Tu, and M. Geva, J. Vac. Sci. Technol. B 18(3), 1476 (2000).

${ }^{15}$ G. Baldassarri H. v. H., M. Bissiri, A. Polimeni, M. Capizzi, M. Fisher, M. Reinhardt, and A. Forchel, Appl. Phys. Lett. 78, 3472 (2001).

${ }^{16}$ L. Wen, F. Bekisli, M. Stavola, W. B. Fowler, R. Trotta, A. Polimeni, M. Capizzi, S. Rubini, and F. Martelli, Phys. Rev. B 81, 233201 (2010).

${ }^{17}$ A. A. Bonapasta, F. Filippone, and G. Mattioli, Phys. Rev. Lett. 98, 206403 (2007).

${ }^{18}$ I. A. Buyanova, M. Izadifard, W. M. Chen, A. Polimeni, M. Capizzi, H. P. Xin, and C. W. Tu, Appl. Phys. Lett. 82, 3662 (2003).

${ }^{19}$ R. Trotta, A. Polimeni, F. Martelli, G. Pettinari, M. Capizzi, L. Felisari, S. Rubini, M. Francardi, A. Gerardino, P. C. M. Christianen, and J. C. Maan, Adv. Mater. 23, 2706 (2011).

${ }^{20}$ Optical Orientation, edited by F. Meier and B. P. Zakharchenya (NorthHolland, Amsterdam, 1984).

${ }^{21}$ The reduction of the SDR ratio at long wavelengths, observed in both asgrown samples, is caused by a contribution of an overlapping PL band that exhibits no SDR effect and is likely related to defects in the epilayers or substrate.

${ }^{22}$ Due to a higher oscillator strength of CB-hh than CB-lh, the overall $P_{P L}$ remains co-polarized with the excitation light when these two transitions strongly overlap. In principle, $P_{P L}$ should be counter-polarized at the longest wavelengths where PL should be dominated by CB-lh. While this can be observed in some samples, it is not so in the samples shown in Figs. 1(a) and 1(b). This is attributed to the presence of the overlapping defectrelated emission (Ref. 21), which exhibits no or even co-polarization with the excitation light such that $P_{P L}$ from CB-lh is strongly compensated or even over compensated leading to the resulting $P_{P L}$.

${ }^{23}$ W. M. Chen, Thin Solid Films 364, 45 (2000). 